\title{
Perceived Roughness of a Grating: Correlation with Responses of Mechanoreceptive Afferents Innervating the Monkey's Fingerpad
}

\author{
K. Sathian, ${ }^{a}$ A. W. Goodwin, K. T. John, and I. Darian-Smith \\ Department of Anatomy, University of Melbourne, Parkville, Victoria 3052, Australia
}

\begin{abstract}
Human subjects scaled gratings of alternating grooves and ridges for perceived roughness. Roughness increased with an increase in groove width and decreased with an increase in ridge width, but the effect of groove width was much greater than the effect of ridge width. In corresponding neurophysiological experiments, the gratings were moved sinusoidally across the receptive fields of single mechanoreceptive afferents innervating the fingerpads of anesthetized monkeys. The measure of response used was the mean cyclic discharge rate (averaged over one cycle of the sinusoid). Slowly adapting afferents (SAs), rapidly adapting afferents (RAs), and Pacinian afferents (PCs) all showed a marked increase in response when groove width increased. An increase in ridge width had no consistent effect on the responses of SAs or RAs but resulted in a small decrease in the response of PCs. The response to a smooth surface differed significantly from the responses to the finer gratings only for the RAs. An alternative measure of response (the number of impulses elicited by each spatial cycle of the grating) increased with an increase in ridge width for all 3 fiber types. Thus, the large effect of groove width on perceived roughness can be accounted for by the mean cyclic discharge rate in the active afferent fibers. The smaller effect of ridge width can be accounted for by the number of impulses per spatial cycle of the grating.
\end{abstract}

Early neurophysiological studies of the spatial and temporal resolving capacities of the tactile system relied on the use of vibrating or indenting probes (Lindblom and Lund, 1966; Talbot et al., 1968; Johnson, 1974). These studies formed an essential first step but were insufficient to explain the more complex events that occur during manual exploration of a textured surface. More recently, various patterned surfaces have been used such as single dots on a smooth surface (LaMotte and Whitehouse, 1986) and matrices of raised dots (Darian-Smith et al., 1980; Johnson and Lamb, 1981; Lamb, 1983). A surface that has proved particularly amenable to quantitative analysis is a grating of alternating grooves and ridges (Darian-Smith and Oke, 1980; Looft, 1986). Two principal advantages of such sur-

\footnotetext{
Received Mar. 14, 1988; revised July 21, 1988; accepted Aug. 25, 1988

This work was supported by a grant from the National Health and Medical Research Council of Australia. We thank Neil Ratcliffe, Colin Clarke, and Alf Medoro for technical assistance.

Correspondence should be addressed to Dr. A. W. Goodwin, Department of Anatomy, University of Melbourne, Grattan Street, Parkville, Victoria 3052, Australia.

a Present address: Department of Anatomy and Neurobiology, Washington University School of Medicine, St. Louis, MO 63110.

Copyright (C) 1989 Society for Neuroscience $0270-6474 / 89 / 041273-07 \$ 02.00 / 0$
}

faces are that they vary in one dimension only, considerably simplifying analysis, and that it is possible to define their spatial characteristics simply and precisely and manufacture them to specification.

The sequence in psychophysical experiments has been the reverse. In one of the earliest descriptions of tactile resolution in humans, Katz utilized gratings formed by winding wire around a pencil (see Krueger, 1970). Natural surfaces such as paper (Meenes and Zigler, 1923) and wool (Binns, 1937) have been used, and emery cloth has been used as a texture, both in human psychophysics (Stevens and Harris, 1962) and more recently to assess the discriminative capacities of monkeys (Carlson, 1984). Detailed studies utilizing vibrating probes did not abound until the 1960s and 1970s (e.g., Verrillo, 1962). Once again, these provided important information, but it was difficult to use the data to elucidate mechanisms of texture perception. Surfaces like emery cloth have the advantage of being readily available in a range of textures, but it is difficult to specify their spatial features and to relate them to human sensation. Therefore, Lederman and Taylor (1972) used metal plates of alternating grooves and ridges to determine the parameters that affected the perception of roughness when subjects rubbed their fingertips back and forth across the gratings. They concluded that perceived roughness increased markedly when groove width widened, and decreased slightly when ridge width widened.

In a series of experiments, Goodwin and Morley recorded the activity of cutaneous mechanoreceptive afferents responding to gratings moved sinusoidally across their receptive fields (Goodwin and Morley, 1987a, b; Morley and Goodwin, 1987). The sinusoidal motion was equivalent to the movement used by humans scanning textured surfaces. They described the responses of the afferents in terms of the spatial period of the grating and the movement characteristics. These data provide a general foundation for explaining the corresponding psychophysical experiments, but in making direct comparisons there are 2 obvious inadequacies. First, when the spatial period of the grating varied, groove width and ridge width covaried with a constant ratio of 7 , so that the separate contribution of these parameters to the response could not be determined. In this paper we independently varied the groove width and the ridge width in order to clucidate the neurophysiological basis of the apparently large difference in their contribution to perceived roughness. The second deficit is that Lederman and Taylor (1972) used a much smaller range of groove widths and ridge widths (only spatial periods of $1.25 \mathrm{~mm}$ or less), and their surfaces differed slightly from ours. Therefore, we extended their roughness measurements to a wider range of groove widths and ridge widths using the same gratings that we used in the neurophysiological experiments. 
Table 1. Dimensions of gratings

\begin{tabular}{|c|c|c|c|}
\hline$G$ & $W$ & $P$ & $G / W$ \\
\hline \multicolumn{4}{|c|}{ Constant ridge: $0.3 \mathrm{~mm}$} \\
\hline 0.44 & 0.31 & 0.74 & 1.4 \\
\hline 0.72 & 0.28 & 1.00 & 2.6 \\
\hline 1.18 & 0.31 & 1.49 & 3.8 \\
\hline 1.73 & 0.27 & 2.00 & 6.5 \\
\hline \multicolumn{4}{|c|}{ Constant ridge: $1.5 \mathrm{~mm}$} \\
\hline 0.18 & 1.59 & 1.77 & 0.11 \\
\hline 0.46 & 1.57 & 2.02 & 0.29 \\
\hline 0.99 & 1.54 & 2.53 & 0.64 \\
\hline 1.52 & 1.54 & 3.06 & 0.98 \\
\hline \multicolumn{4}{|c|}{ Constant groove: $0.25 \mathrm{~mm}$} \\
\hline 0.24 & 0.76 & 1.00 & 0.32 \\
\hline 0.20 & 1.06 & 1.27 & 0.19 \\
\hline 0.25 & 1.80 & 2.05 & 0.14 \\
\hline 0.25 & 2.75 & 3.00 & 0.092 \\
\hline \multicolumn{4}{|c|}{ Constant groove: $0.5 \mathrm{~mm}$} \\
\hline 0.44 & 0.31 & 0.74 & 1.4 \\
\hline 0.44 & 0.56 & 1.00 & 0.78 \\
\hline 0.51 & 1.04 & 1.54 & 0.49 \\
\hline 0.46 & 1.57 & 2.02 & 0.29 \\
\hline 0.48 & 2.58 & 3.06 & 0.19 \\
\hline \multicolumn{4}{|c|}{ Constant groove: $1 \mathrm{~mm}$} \\
\hline 0.96 & 0.16 & 1.13 & 5.9 \\
\hline 0.97 & 0.29 & 1.25 & 3.4 \\
\hline 0.94 & 0.57 & 1.51 & 1.6 \\
\hline 0.95 & 1.05 & 2.00 & 0.90 \\
\hline 0.99 & 1.54 & 2.53 & 0.64 \\
\hline 0.97 & 2.00 & 2.97 & 0.49 \\
\hline \multicolumn{4}{|c|}{ Constant groove: $1.25 \mathrm{~mm}$} \\
\hline 1.28 & 0.11 & 1.39 & 11. \\
\hline 1.27 & 0.25 & 1.52 & 5.0 \\
\hline 1.27 & 0.48 & 1.75 & 2.6 \\
\hline 1.27 & 1.01 & 2.28 & 1.2 \\
\hline 1.24 & 1.77 & 3.01 & 0.70 \\
\hline \multicolumn{4}{|c|}{ Constant groove: $1.5 \mathrm{~mm}$} \\
\hline 1.45 & 0.21 & 1.66 & 6.8 \\
\hline 1.42 & 0.58 & 2.00 & 2.4 \\
\hline 1.47 & 0.79 & 2.25 & 1.9 \\
\hline 1.46 & 1.05 & 2.51 & 1.4 \\
\hline 1.46 & 1.54 & 3.00 & 0.95 \\
\hline \multicolumn{4}{|c|}{ Constant groove: $2 \mathrm{~mm}$} \\
\hline 1.98 & 0.13 & 2.10 & 15. \\
\hline 2.00 & 0.28 & 2.28 & 7.2 \\
\hline 2.00 & 0.56 & 2.56 & 3.6 \\
\hline 2.00 & 0.84 & 2.83 & 2.4 \\
\hline 2.02 & 1.06 & 3.09 & 1.9 \\
\hline
\end{tabular}

$G, W$ and $P$ give the values (in $\mathrm{mm}$ ) of the groove width, ridge width, and spatial period, respectively. $G / W$ gives the groove width to ridge width ratio. Ridge width was nominally constant in the first 2 blocks, and groove width in the remaining 6 blocks.

\section{Materials and Methods}

Neurophysiological studies. Similar methods have been used by us previously (Goodwin and Morley, 1987a), and only a brief description is given here. Functionally single mechanoreceptive afferents, innervating the digital pads, were microdissected from median nerves in anesthetized macaques. Five $M$. nemestrina and $3 M$. fascicularis, weighing between 2 and $5 \mathrm{~kg}$ were used. The monkeys were tranquilized by intramuscular injection of ketamine hydrochloride and anesthetized by intravenous injection of sodium pentobarbital. Anesthesia was maintained by intraperitoneal injections of sodium pentobarbital. Body temperature was monitored with a rectal thermometer and was maintained around $37^{\circ} \mathrm{C}$ with a heating blanket. Antibiotic cover was provided with amoxicillin sodium $(18 \mathrm{mg} / \mathrm{kg}$ administered intramuscularly at $8 \mathrm{hr}$ intervals). At the end of the experiment, the animal was given intramuscular penicillin $(225 \mathrm{mg}$ benzathine penicillin plus $150 \mathrm{mg}$ procaine penicillin plus $90 \mathrm{mg}$ potassium penicillin) and allowed to recover.

The center of the receptive field of the isolated afferent was determined using a set of graded von Frey hairs. Only those afferents that had receptive fields on the central portion of the fingerpad were used. Afferents were characterized as slowly adapting afferents (SAs), rapidly adapting afferents (RAs) or Pacinian afferents (PCs) based on their responses to static indentation of the receptive field and on their vibratory tuning curves (Talbot et al., 1968; Goodwin and Morley, 1987a). The PCs had a wider receptive field than the RAs or SAs, but each of those included in this study had a clearly identifiable region of maximum sensitivity that was located on a fingerpad. Thirteen SAs, 17 RAs, and 7 PCs were studied.

The fingerpad innervated by the afferent was stimulated by a grating of alternating grooves and ridges moved sinusoidally over the receptive field. The axis of motion was at right angles to the long axis of the finger. The finger was securely fixed to a hand holder using modeling clay, preventing lateral movement, but the fingerpad was free to move normally during grating stimulation. The grating (nylon with a steel backing) was held magnetically on a stimulator arm that moved sinusoidally with a peak-to-peak amplitude of $80 \mathrm{~mm}$. The period of the sinusoidal motion was $3.14 \mathrm{sec}$, resulting in a peak speed of $80 \mathrm{~mm} / \mathrm{sec}$. The finger was indented $1 \mathrm{~mm}$ into the grating, which, on contact, was tangential to the fingerpad at the receptive field center. Analog signals of the position of the grating and the contact force between the finger and the grating were sampled by an analog-to-digital converter. The sinusoidal motion of the grating was further encoded by an optical system that generated a TTL pulse for every cycle and TTL pulses for every $6^{\circ}$ of the cycle. A laboratory computer was used to record these signals as well as the time of occurrence of action potentials from the afferents.

As pointed out in the introductory remarks, the aim of the present study was to examine, independently, the effect of the groove widths and of the ridge widths of the gratings on the afferents' discharges. Thus, the protocol consisted of 2 subsets. In the first subset, the ridge width was held constant at 1 of 2 possible nominal values and groove width was varied. In the second subset, groove width was held constant at 1 of 6 possible nominal values and ridge width was varied. Table 1 gives the actual dimensions of the gratings. For all gratings the grooves were sufficiently deep that neither the monkeys' fingerpads nor those of the human subjects contacted the bottoms of the grooves.

Psychophysical studies. Four naive subjects, ranging in age from 20 to 30 years, with callus-free fingerpads, scaled the gratings for perceived roughness using the technique of magnitude estimation. The gratings (100 mm long and $20 \mathrm{~mm}$ wide) were held magnetically on a block, clamped rigidly to a table; stops on each end of the block prevented the finger from running off the surface. Subjects were required to assess the gratings by rubbing any one fingerpad back and forth across the surface. All subjects chose their right index finger, and no restrictions were placed on their choice of movement patterns. They were seated comfortably behind a curtain so that the surfaces could not be seen by them.

There were 2 independent series for each subject. In the first series, the grating ridge width was held constant, at a nominal value of 0.3 or $1.5 \mathrm{~mm}$, and roughness was estimated as a function of groove width. These 8 surfaces (see Table 1), plus a perfectly smooth surface, were presented in a pseudo random order. In the second series, the groove width was held constant at a nominal value of 1 or $2 \mathrm{~mm}$, and the 11 surfaces (see Table 1) were scaled for roughness as a function of ridge width. Subjects were unaware of the number of gratings in each series as well as the difference between the 2 series.

Each subject had one experimental session per day lasting about 30$45 \mathrm{~min}$. The first few sessions were used for familiarization, and these data were excluded from the analysis. A further 3-4 sessions were required to ensure a sufficient number of presentations of each grating for statistical analysis. At the beginning of each session, and at regular intervals, 2 reference surfaces were presented, and the subjects were told that these would be representative of one of the smoothest and one of the roughest surfaces in the series. They were at liberty to choose any numbers to represent their roughness estimate. 
Subject $S_{1}$
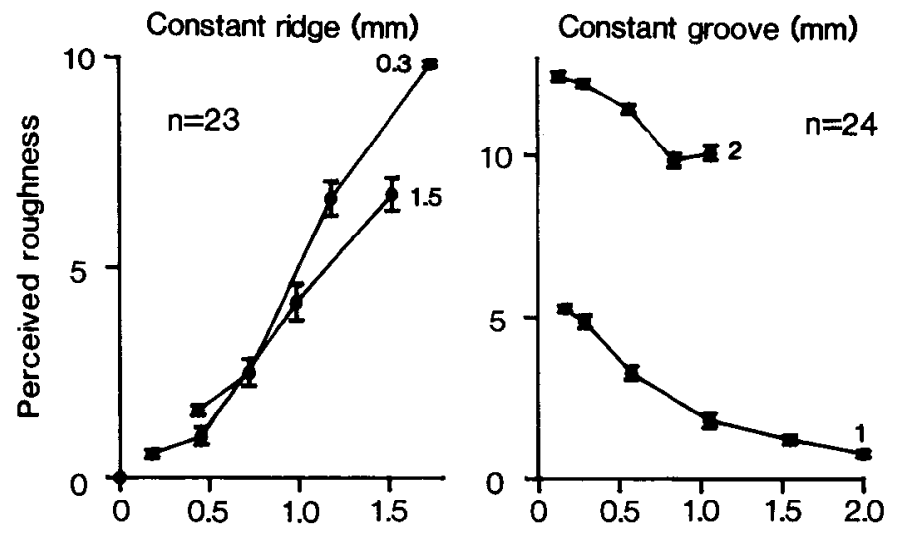

Subject $\mathrm{S}_{2}$
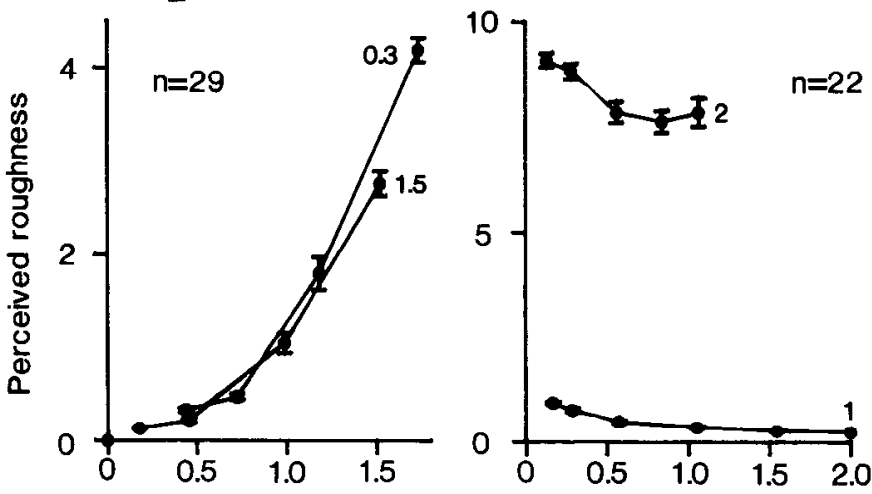

Subject $\mathrm{S}_{3}$
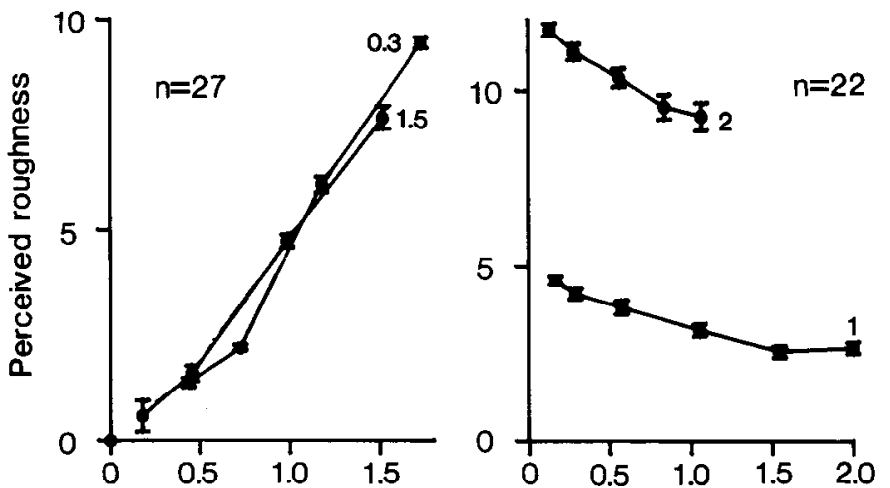

Subject $\mathrm{S}_{4}$

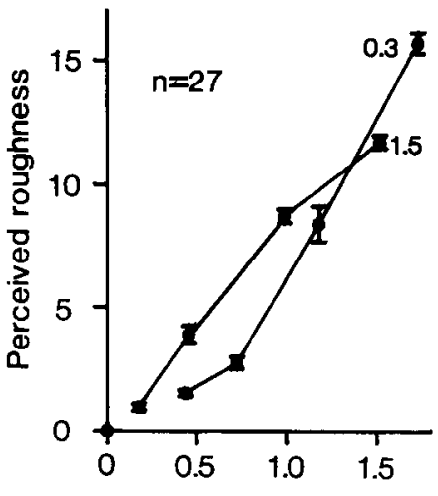

Groove width $(\mathrm{mm})$

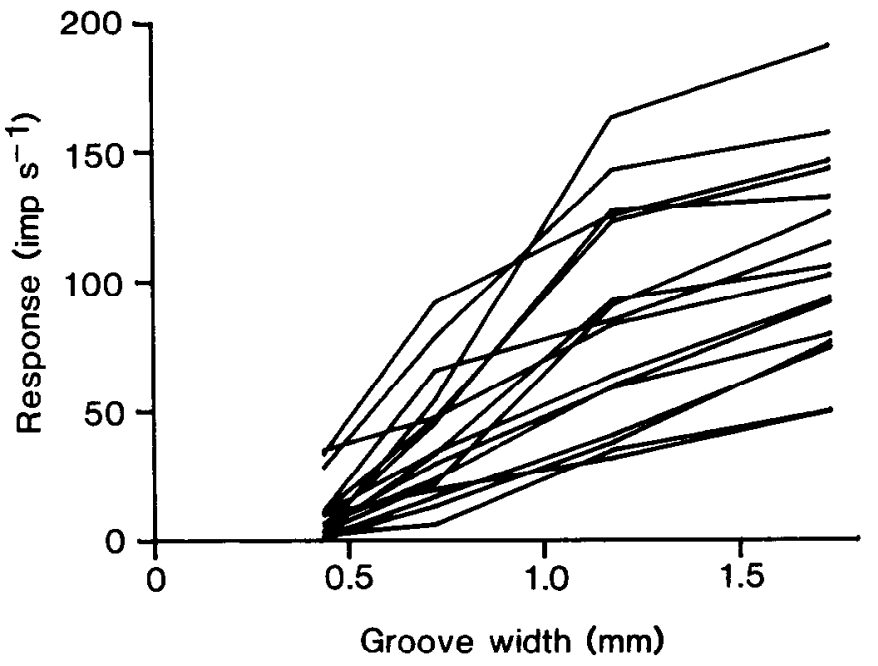

Figure 2. Mean cyclic responses of 13 SAs as a function of groove width. Ridge width was held constant at $0.3 \mathrm{~mm}$.

\section{Results}

\section{Psychophysical experiments}

Figure 1 (left) illustrates the results of the 4 subjects scaling gratings for perceived roughness in the 2 blocks for which ridge width remained constant. For each grating presented, the mean magnitude estimate and the SEM have been plotted. All 4 subjects perceived roughness increasing nearly linearly as the groove width increased (see Table 2). The differing ordinate scales in the 4 graphs result from the individual subjects' choices of magnitude estimate scales. In all trials in which the smooth surface was presented, all subjects identified it correctly and designated it as having "zero" roughness.

All subjects found the series of constant groove widths more difficult to scale than the series of constant ridge widths. Figure 1 (right) represents the results from this series. All 4 subjects scaled the gratings as a monotonic function of ridge width. As the ridge widened, 3 subjects reported decreasing roughness, while the fourth subject $\left(S_{4}\right)$ reported increasing roughness. The low SEs are indicative of the consistency of the magnitude estimates despite the subjects' apparent difficulties in scaling this series. In general, the functions are not linear (see Table 2), but in all cases the regression slopes are significantly different from zero ( $t$ test, $p<0.005$ ). The marked effect of groove width is evident in the curves for all 4 subjects, as the gratings with a constant groove width of $2 \mathrm{~mm}$ were consistently assigned a higher magnitude estimate than the gratings with a constant groove width of $1 \mathrm{~mm}$. In contrast, such a clear-cut distinction does not exist between the ridge widths of 0.3 and $1.5 \mathrm{~mm}$ in the constant ridge width series (Fig. 1, left).

Comparison of the slopes of the lines in Figure 1 (left) with

Figure 1. Perceived roughness (mean \pm 1 SEM) as a function of groove width and ridge width for 4 subjects. Left, Ridge width held constant at 0.3 or $1.5 \mathrm{~mm}$, and groove width varied. Roughness for the smooth surface is shown at a groove width of $0 \mathrm{~mm}$. Right, Groove width held constant at 1 or $2 \mathrm{~mm}$, and ridge width varied. Number of observations at each point is given by $n$. Note different ordinate scales for different subjects. 
Table 2. Linear-regression coefficients for psychophysics

\begin{tabular}{|c|c|c|c|c|c|c|c|c|}
\hline \multirow[b]{2}{*}{ Const. } & \multicolumn{2}{|l|}{$S_{1}$} & \multicolumn{2}{|l|}{$\mathbf{S}_{2}$} & \multicolumn{2}{|l|}{$\mathbf{S}_{3}$} & \multicolumn{2}{|l|}{$\mathrm{S}_{4}$} \\
\hline & $r^{2}$ & $b$ & $r^{2}$ & $b$ & $r^{2}$ & $b$ & $r^{2}$ & $b$ \\
\hline$W=0.3$ & 0.85 & 6.7 & 0.82 & 3.1 & 0.94 & 6.5 & 0.84 & 11.3 \\
\hline$W=1.5$ & 0.73 & 4.8 & 0.77 & 2.0 & 0.89 & 5.4 & 0.88 & 8.0 \\
\hline$G=1$ & 0.74 & -2.5 & 0.62 & -0.4 & 0.46 & -1.1 & 0.68 & 2.1 \\
\hline$G=2$ & 0.55 & -2.9 & 0.16 & -1.5 & 0.30 & -2.6 & 0.47 & 6.9 \\
\hline
\end{tabular}

Coefficients of determination $\left(r^{2}\right)$ and slopes of regression lines $(b)$ for the 4 subjects (S). Either the ridge width $(W, \mathrm{~mm})$ or the groove width $(G, \mathrm{~mm})$ was held constant as indicated in the left column.

those in Figure 1 (right), for each subject, suggests that changes in groove width have a greater effect on perceived roughness than equal changes in ridge width. However, since the data for the 2 figures were collected in independent series, the subjects may have changed their "scales," invalidating direct comparison of slopes. To circumvent this problem, both slopes were estimated from Figure 1 (right). The slope with groove width was estimated from the difference in roughness for the 1 and 2 $\mathrm{mm}$ groove widths, averaged at 3 ridge widths. For the 4 subjects, the average magnitudes of the slopes (roughness per $\mathrm{mm}$ ) for groove width were $7.9,7.6,6.5$, and 12.4 ; and for ridge width, $2.7,1.0,1.9$, and 4.5 , respectively.

\section{Responses of cutaneous mechanoreceptive afferents}

As shown previously by Goodwin and Morley (1987a), all afferents responded in synchrony with a grating moved sinusoidally over the receptive field. The profile of the cycle histogram

Table 3. Linear-regression coefficients for afferent responses

Const.

$r^{2}$

$b$

SAs: constant ridge

$W=0.3$

0.63

0.60

$W=1.5$

0.34

0.23

RAs: constant ridge

$W=0.3$

0.88

0.88

$W=1.5$

0.80

0.59

PCs: constant ridge

$\boldsymbol{W}=0.3$

0.71

0.47

$W=1.5$

0.77

0.54

SAs: constant groove

$G=1$

0.10

0.09

$G=1.25$

0.07

0.07

$G=1.5$

0.02

$-0.04$

$G=2$

0.03

$-0.13$

RAs: constant groove

$G=0.25$

0.14

0.04

$G=0.5$

0.21

0.11

$G=1$

$G=1.25$

0.01

0.10

$-0.02$

$-0.08$

$-0.11$

$-0.12$

$G=2$

0.07

$-0.07$

0.07

$G=0.5$

0.52

$-0.25$

$=1$

0.32

$-0.35$

Coefficients of determination $\left(r^{2}\right)$ and slopes of regression lines $(b)$ for the 3 afferent types. Either the ridge width $(W, \mathrm{~mm})$ or the groove width $(G, \mathrm{~mm})$ was held constant as indicated in the left column. varied for the different afferents and different stimulus conditions (Morley and Goodwin, 1987). One simple measure of an afferent's response is the mean cyclic response (average discharge rate over one cycle of grating movement), and this measure is used here.

Figure 2 shows the responses of 13 SAs when the ridge width was held constant at $0.3 \mathrm{~mm}$. All afferents behaved similarly in that they all showed a monotonic increase in discharge rate with an increase in groove width. However, at any groove width, absolute rates showed a wide scatter due to variation in sensitivity among the afferents. To study the consistency (and statistical reliability) of the relationship between groove width and response, the effect of the sensitivity of individual afferents was removed by normalizing responses prior to pooling. This was accomplished by dividing the responses of each afferent by a normalizing factor that was the average response to the 14 gratings in the blocks, groove $=1.25 \mathrm{~mm}$, groove $=2 \mathrm{~mm}$, and ridge $=0.3 \mathrm{~mm}$ (Table 1$)$.

The mean normalized responses of the SA population are shown in Figure $3 A$ (left) for both blocks at constant ridge width. Because the intrinsic relationship between response and groove width was the same for all SAs, regardless of their sensitivity, the SEs in Figure $3 A$ (left) are small. The small SEs in the remaining curves in Figure 3 verify that the same argument holds for all 3 afferent types under all stimulus conditions. Solid arrowheads on the ordinate axis indicate the response to the smooth surface and open arrowheads the spontaneous activity in the absence of any stimulus. Figure $3 B$ (left) and $3 C$ (left) shows the same data for the RAs and PCs, respectively. For the RAs and PCs, the normalizing factor was the average response to the 11 gratings in the blocks, groove $=1 \mathrm{~mm}$ and groove $=$ $2 \mathrm{~mm}$. For all 3 afferent types, the striking observation is that the response increased markedly when groove width increased, but there is little difference between the curves when the ridge width was $0.3 \mathrm{~mm}$ and when it was $1.5 \mathrm{~mm}$. The results of linear-regression analysis of the curves are given in Table 3. The distinction between the responses to the smooth surface and the responses to the gratings with the narrowest groove widths differed for the 3 fiber types. For the RAs, at both ridge widths, the response to the smooth surface was significantly less than the responses to the gratings with the narrowest grooves. For the SAs, the response to the smooth surface did not differ from responses to the narrowest grooves at either ridge width. For the PCs, the response to the smooth surface was significantly less than the response to the narrowest groove at a ridge width of $0.3 \mathrm{~mm}$, but did not differ at a ridge width of $1.5 \mathrm{~mm}$. These comparisons were made with a $t$ test at the level $p<0.05$.

The right column of Figure 3 shows the responses of the 3 groups of afferents as a function of ridge width when groove width was held constant. For the RAs, 6 groove widths were 


\section{A. Slowly adapting afferents}

Constant ridge $(\mathrm{mm})$

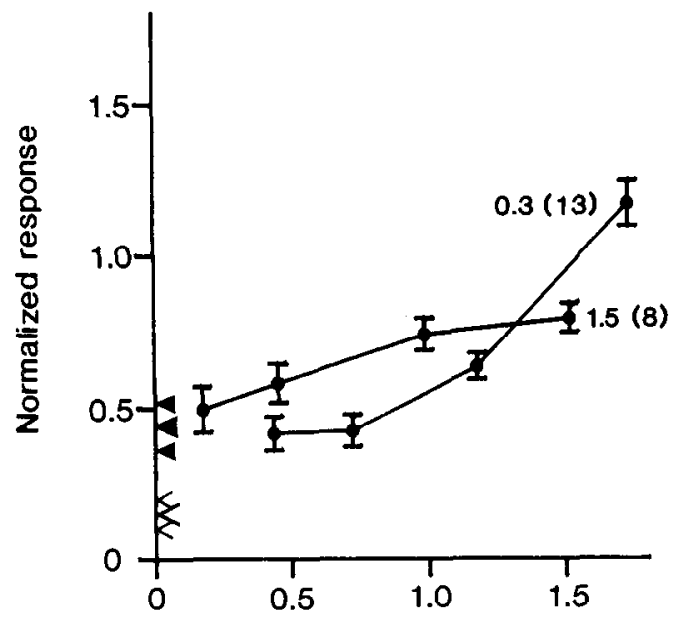

\section{B. Rapidly adapting afferents}

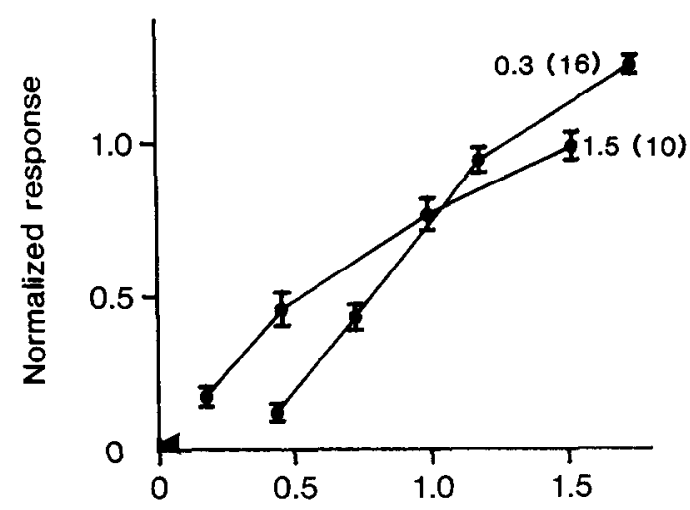

\section{Pacinian afferents}

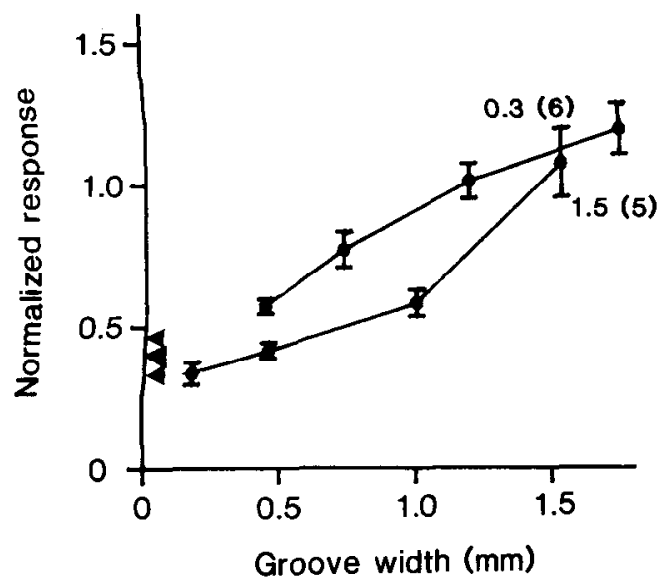

\section{Constant groove $(\mathrm{mm})$}

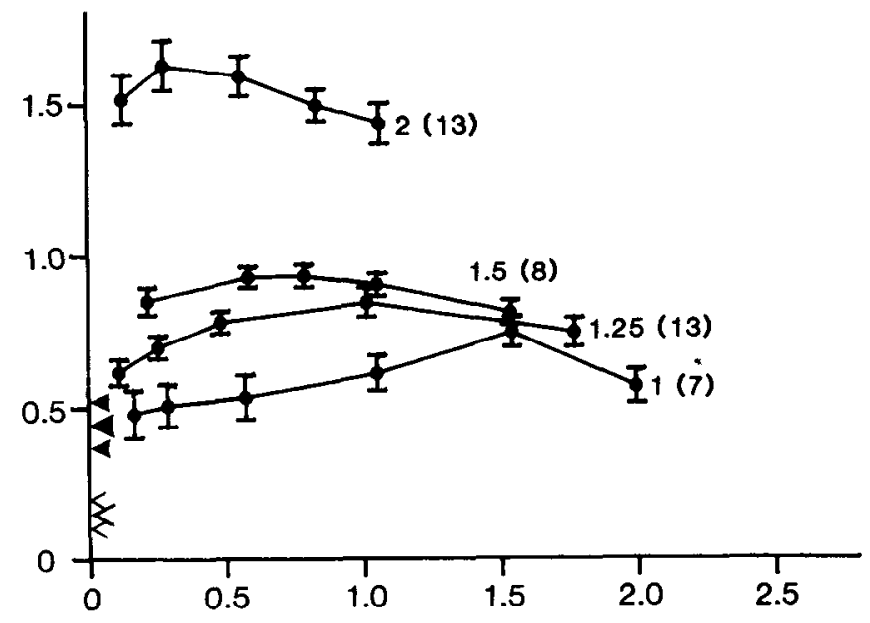

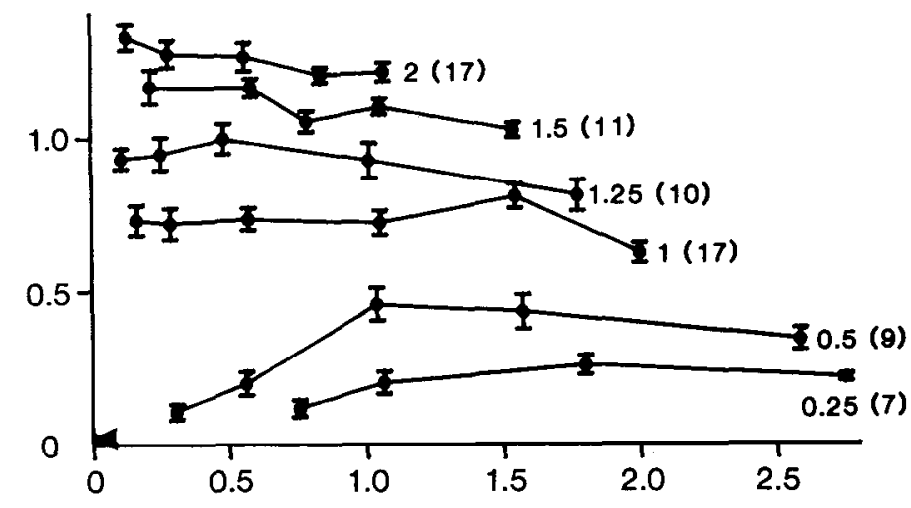

Figure 3. Normalized mean cyclic responses of the 3 fiber types. In the left column, the ridge width was held constant at 0.3 or $1.5 \mathrm{~mm}$, and responses are plotted as a function of groove width. In the right column, groove width was held constant at the values indicated, and responses are plotted as a function of ridge width. Responses are displayed as means \pm 1 SEM, and numbers in parentheses show the number of afferents in the pool. Solid arrowheads indicate responses to a smooth surface (mean \pm 1 SEM) and, for the SAs, open arrowheads indicate spontaneous activity in the absence of any stimulus. Note that for RAs, the SEM for the smooth surface is negligible. 


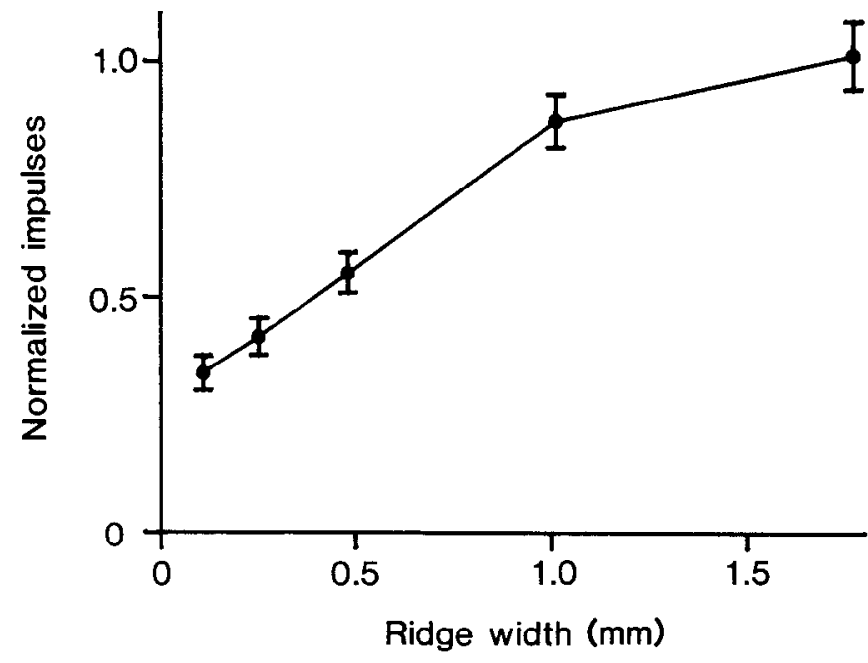

Figure 4. Responses, during the central $42^{\circ}$ of each half-cycle (when speed is approximately constant), for the $13 \mathrm{SAs}$ in our sample. The mean number of impulses elicited by each grating spatial cycle have becn normalized and averaged over the population. Mean \pm 1 SEM are shown. Groove width was held constant at $1.25 \mathrm{~mm}$.

used. For the SAs, the 2 narrowest grooves were not used since responses to these were indistinguishable from responses to a smooth surface. Only 3 groove widths were used for the PCs. The overwhelming impression, from the right column of Figure 3 , is that ridge width had only a minor effect on the responses of all 3 afferent classes. This is borne out by the results of linearregression analysis given in Table 3 . On the other hand, each constant ridge width line is clearly separated from its neighbors, reflecting the clear effect of groove width (which is also seen in the left column). For the SAs and RAs, response was a decreasing function of ridge width for wide grooves and an increasing function of ridge width for narrow grooves. This results in the crossover point in Figure $3 A$ (left) and $3 B$ (left) where ridge width had no effect, corresponding to a horizontal line in Figure $3 \mathrm{~A}$ (right) and $3 B$ (right), respectively. Thus, for SAs and RAs, the ridge effect was minor and was variable with groove width. For the PCs, all groove widths resulted in responses decreasing as a function of ridge width (although the effect was only slight).

The correspondence between the neural responses in the left column of Figure 3 and the human responses in the left column of Figure 1 is obvious. In contrast, the relationship between the right column of Figure 3 and the right column of Figure 1 is tenuous. An alternative measure of response results from the fact that, during the central $42^{\circ}$ of each half-cycle of movement, the grating speed is constant to within $6.6 \%$ of its peak value; here, afferent discharges are phase-locked to the temporal occurrence of grating spatial cycles (Morley and Goodwin, 1987). The number of impulses elicited by each spatial cycle of the grating varies with the parameters of the grating. Figure 4 shows this measure of response, for the SAs, as a function of ridge width; groove width was held constant at $1.25 \mathrm{~mm}$. Responses were normalized before pooling. Figure 4 corresponds to the groove $=1.25 \mathrm{~mm}$ line in Figure $3 A$ (right); comparison shows that, while increasing ridge width did not have a consistent effect on the mean cyclic response, it resulted in a clear monotonic increase in the number of impulses per grating spatial cycle. The same result held for all groove widths for all 3 fiber types, as can be seen in Figure 1 of the following paper (Goodwin et al.,
1989), where this measure of response is pursued in detail in a different context.

\section{Discussion}

Human perception of roughness

Increasing the groove width of a grating resulted in a marked increase in the subjects' perceived roughness. When the ridge width changed, a definite change in roughness was perceived, but the effect was smaller than that produced by equal changes in groove width (by a factor of about 3). Moreover, for 3 of the subjects, an increase in ridge width resulted in a decrease in roughness. These results extend Lederman and Taylor's (1972) data on a narrower range of gratings and are consistent with their observations. The smooth surface differed from our other surfaces in that there was no uncertainty in identifying it (0 SE). Morley et al. (1983) increased the spatial period of their gratings while maintaining the ratio of groove width to ridge width constant at 9 , and they found an increase in perceived roughness. This is expected from our data, as the effect of the increase in groove width would dominate the opposite but less sensitive effect of the increase in ridge width, which in any case would be 9 times smaller.

The fourth subject showed approximately the same sensitivity to changes in ridge width but interpreted an increase in ridge width as an increase in roughness. Thus, the "sensory" neural signals in this subject were as sensitive to changes in ridge width as in the other subjects (and presumably differences in ridge width could be discriminated as effectively), but the "interpretation" of roughness was reversed. The fact that this subject's interpretation of roughness was the same as the other subjects' when groove width changed could result from different cues being used in the 2 tasks (see below). Such observations highlight the difficulty of interpreting a subject's reported perceptions in psychophysical experiments.

\section{Correlation of perception and afferent fiber responses}

The responses of afferent fibers could be studied only in anesthetized monkeys, necessitating passive stimulation. In order to compare these responses to our psychophysical results, we chose a stimulus motion that closely resembles the natural movements used by humans. Thus, the motion was sinusoidal with a peak speed of $80 \mathrm{~mm} / \mathrm{sec}$, which is in the range normally used by humans in such tasks (Lederman, 1974; Morley et al., 1983).

The mean cyclic response provides a direct measure of the activity of a fiber. This simple time average is easy to compute not only for the experimenter but presumably also for the brain. In the constant ridge width series, an increase in groove width resulted in an increase in the mean cyclic response of all 3 classes of afferents. Thus, this measure contains sufficient information to account for our subjects' scaling performance. The 3 populations differed in their ability to resolve the smooth surface from the finer gratings. On the basis of mean cyclic response, only the RAs can account for the subjects' ability to distinguish the smooth surface consistently. This parallels the observation of LaMotte and Whitehouse (1986) that only RAs can detect the presence of a small raised dot on a smooth surface stroked across the fingerpad.

Increases in ridge width clicited no consistent changes in the mean cyclic rates of the SAs or the RAs but did result in a small decrease in the rates of the PCs. Thus, on the basis of mean cyclic response, only the PCs can explain the dependence of perceived roughness on ridge width, and even this relationship 
is not entirely convincing. However, there are other less direct measures of response available in the primary afferent discharge. During the portion of the movement cycle when speed is approximately constant, the afferent impulses are phase-locked to the occurrence of successive spatial cycles of the grating. A second measure of response is the number of phase-locked impulses per grating spatial cycle. This measure clearly increases when ridge width increases (for all 3 fiber types) and could easily provide the information necessary to explain the human performance. A third possibility results from the fact that the number of phase-locked bursts decreases as the ridge width increases (with groove width constant).

With a multidimensional stimulus as complex as a moving grating, the neural discharge contains several candidate codes for each of the stimulus parameters. At each level of the nervous system we can identify the candidate codes and reject those that are not feasible (Perkel and Bullock, 1968). During different tasks subjects may use different codes. For example, they may well have used the mean cyclic discharge rate when scaling the blocks with constant ridge width, but the number of impulses per grating spatial cycle when scaling the blocks with constant groove width. The strategy for judging an attribute as complex as roughness cannot be considered invariant; it may vary from subject to subject and even within subjects for differcnt tasks.

Since mean cyclic responses are a function of groove width but not ridge width, it is likely that the effects reported by Goodwin and Morley (1987a) to changes in the spatial period of a grating simply reflect the changes in groove width. However, such a simple statement cannot be made in relation to the magnitude of the phase-locked response, which is a function of both groove width and ridge width, nor is it easy to hypothesize why groove width and ridge width have different effects, as these effects are different for different response measures. Meaningful comparisons can be made at the quantitative level only if a complete mathematical model of responses is available; this is the subject of the following paper (Goodwin et al., 1989).

\section{Responses of Pacinian afferents}

The responses of the SAs and RAs in our data are entirely consistent with those reported by Goodwin and Morley (1987a), but there are differences in the PC responses. All our PCs behaved in the same way, increasing in response with an increase in groove width, and they behaved quite similarly to RAs except for the small decrease in mean cyclic response with increasing ridge width. In contrast, Goodwin and Morley reported considerable variability among their PCs, and, on average, mean cyclic responses did not increase significantly with increasing spatial period. We believe that the differences result from differences in the location of the PCs. Goodwin and Morley recorded from PCs that "were most sensitive to stimuli on one finger (rather than other parts of the hand) and usually were most sensitive to stimuli on the 2 most distal phalanges of that finger." We were more demanding, and restricted our sample to PCs with receptive fields that unequivocally had their most sensitive point on the distal phalanx (usually the fingerpad). In both studies vibratory tuning curves confirmed the classification of the receptors (Talbot et al., 1968). It is not surprising that more proximally located PCs cannot resolve spatial details at the fingerpad. The important point, however, which is not usually stressed, is that the PCs located in the fingerpad behave more similarly to RAs and have greater capacities to discriminate spatial detail than the more proximally located receptors that are usually studied.

\section{References}

Binns, H. (1937) Visual and tactual 'judgement' as illustrated in a practical experiment. Br. J. Psychol. 27: 404-410.

Carlson, M. (1984) Development of tactile discrimination capacity in Macaca mulatta. I. Normal infants. Dev. Brain Res. 16: 69-82.

Darian-Smith, I., and L. E. Oke (1980) Periphcral neural representation of the spatial frequency of a grating moving across the monkey's finger pad. J. Physiol. (Lond.) 309: 117-133.

Darian-Smith, I., I. Davidson, and K. O. Johnson (1980) Peripheral neural representation of spatial dimensions of a textured surface moving across the monkey's finger pad. J. Physiol. (Lond.) 309: 135-146.

Goodwin, A. W., and J. W. Morley (1987a) Sinusoidal movement of a grating across the monkey's fingerpad: Representation, of grating and movement features in afferent fiber responses. J. Neurosci. 7 : $2168-2180$.

Goodwin, A. W., and J. W. Morley (1987b) Sinusoidal movement of a grating across the monkey's fingerpad: Effect of contact angle and force of the grating on afferent fiber responses. J. Neurosci. 7:21922202.

Goodwin, A. W., K. T. John, K. Sathian, and I. Darian-Smith (1989) Spatial and temporal factors determining afferent fiber responses to a grating moving sinusoidally over the monkey's fingerpad. J. Neurosci. 9: 1280-1293

Johnson, K. O. (1974) Reconstruction of population response to a vibratory stimulus in quickly adapting mechanoreceptive afferent fiber population innervating glabrous skin of the monkey. J. Neurophysiol. 35: 48-72.

Johnson, K. O., and G. D. Lamb (1981) Neural mechanisms of spatial tactile discrimination: Neural patterns evoked by braille-like dot patterns in the monkey. J. Physiol. (Lond.) 310: 117-144.

Krueger, L. E. (1970) David Katz's Der Aufbau der Tastwelt (The world of touch): A synopsis. Percept. Psychophys. 7: 337-341.

Lamb, G. D. (1983) Tactile discrimination of textured surfaces: Periphcral neural coding in the monkey. J. Physiol. (Lond.) 338: 567587.

LaMotte, R. H., and J. Whitehouse (1986) Tactile detection of a dot on a smooth surface: Peripheral neural events. J. Neurophysiol, 56 : $1109-1128$.

Lederman, S. J. (1974) Tactile roughness of grooved surfaces: The touching process and effects of macro- and microsurface structure. Percept. Psychophys. 16: 385-395.

Lederman, S. J., and M. M. Taylor (1972) Fingertip force, surface geometry, and the perception of roughness by active touch. Percept. Psychophys. 12: 401-408.

Lindblom, U., and L. Lund (1966) The discharge from vibrationsensitive receptors in the monkey foot. Exp. Neurol. 15: 401-417.

Looft, F. J. (1986) Response of cat cutaneous mechanoreceptors to punctate and grating stimuli. J. Neurophysiol. 56: 208-220.

Meenes, M., and M. J. Zigler (1923) An experimental study of the perceptions of roughness and smoothness. Am. J. Psychol. 34: 542549.

Morley, J. W., and A. W. Goodwin (1987) Sinusoidal movement of a grating across the monkey's fingerpad: Temporal patterns of afferent fiber responses. J. Neurosci. 7: 2181-2191.

Morley, J. W., A. W. Goodwin, and I. Darian-Smith (1983) Tactile discrimination of gratings. Exp. Brain Res. 49: 291-299.

Perkel, D. H., and T. H. Bullock (1968) Neural coding. Neurosci. Res. Prog. Bull. 6: 221-348.

Stevens, S. S., and J. R. Harris (1962) The scaling of subjective roughness and smoothness. J. Exp. Psychol. 64: 489-494.

Talbot, W. H., I. Darian-Smith, H. H. Kornhuber, and V. B. Mountcastle (1968) The sense of flutter-vibration: Comparison of the human capacity with response patterns of mechanoreceptive afferents from the monkey hand. J. Neurophysiol. 31: 301-334.

Verrillo, R. T. (1962) Investigation of some parameters of the cutaneous threshold for vibration. J. Acoust. Soc. Am. 34: 1768-1773. 